Journal for

.... ImmunoTherapy of Cancer

\section{CD4+ T cell and M2 macrophage infiltration predict dedifferentiated liposarcoma patient outcomes}

To cite: Schroeder BA, LaFranzo NA, LaFleur BJ, et al. CD4+ T cell and M2 macrophage infiltration predict dedifferentiated liposarcoma patient outcomes. Journal for ImmunoTherapy of Cancer 2021;9:e002812. doi:10.1136/ jitc-2021-002812

- Additional supplemental material is published online only. To view, please visit the journal online (http://dx.doi.org/10. 1136/jitc-2021-002812).

Accepted 26 July 2021
Check for updates

(C) Author(s) (or their employer(s)) 2021. Re-use permitted under CC BY-NC. No commercial re-use. See rights and permissions. Published by BMJ.

For numbered affiliations see end of article.

Correspondence to

Dr Seth M Pollack;

Seth.pollack@northwestern.edu

\section{ABSTRACT}

Background Dedifferentiated liposarcoma (DDLPS) is one of the most common soft tissue sarcoma subtypes and is devastating in the advanced/metastatic stage. Despite the observation of clinical responses to $P D-1$ inhibitors, little is known about the immune microenvironment in relation to patient prognosis.

Methods We performed a retrospective study of 61 patients with DDLPS. We completed deep sequencing of the T-cell receptor (TCR) $\beta$-chain and RNA sequencing for predictive modeling, evaluating both immune markers and tumor escape genes. Hierarchical clustering and recursive partitioning were employed to elucidate relationships of cellular infiltrates within the tumor microenvironment, while an immune score for single markers was created as a predictive tool.

Results Although many DDLPS samples had low TCR clonality, high TCR clonality combined with low T-cell fraction predicted lower 3-year overall survival $(p=0.05)$. Higher levels of CD14 + monocytes $(p=0.02)$ inversely correlated with 3-year recurrence-free survival (RFS), while CD4 + T-cell infiltration $(p=0.05)$ was associated with a higher RFS. Genes associated with longer RFS included $P D-1(\mathrm{p}=0.003)$, ICOS $(\mathrm{p}=0.006), B T L A(\mathrm{p}=0.033)$, and CTLA4 $(\mathrm{p}=0.02)$. In a composite immune score, CD4 + Tcells had the strongest positive predictive value, while CD14 + monocytes and M2 macrophages had the strongest negative predictive values.

Conclusions Immune cell infiltration predicts clinical outcome in DDLPS, with CD4 + cells associated with better outcomes; CD14 + cells and M2 macrophages are associated with worse outcomes. Future checkpoint inhibitor studies in DDLPS should incorporate immunosequencing and gene expression profiling techniques that can generate immune landscape profiles.

\section{INTRODUCTION}

Dedifferentiated liposarcoma (DDLPS) is a devastating and often aggressive liposarcoma (LPS) subtype with increasing incidence. ${ }^{1}$ Recent approvals of eribulin and trabectedin have given hope for improved outcomes in the advanced/metastatic setting, but median survival remains less than 3 years. ${ }^{2-4}$ Although DDLPS arises de novo in a majority of cases, approximately $10 \%$ arise from the more indolent well-differentiated liposarcoma (WDLPS)/atypical lipomatous tumor. ${ }^{5}$ WDLPS and DDLPS both frequently harbor chromosome-12 abnormalities, including amplification of chromosome 12q14-15, which includes $M D M 2$ and $C D K 4 .{ }^{67}$ The detection of $M D M 2$ amplification is an important tool for diagnostic confirmation of these neoplasms. MDM2 has been considered a target for novel therapeutic approaches, ${ }^{8}$ and direct inhibition of $C D K 4$ can be effective for some patients. ${ }^{910}$ Given their common molecular pathogenesis and the well-characterized progression from WDLPS to DDLPS in a subset of patients, these are often considered together as part of a single disease entity, well/dedifferentiated liposarcoma (WD/DD LPS). ${ }^{11}$

Significant challenges remain in using programmed cell death protein 1 (PD-1) inhibitors for the treatment of DDLPS. Of the initial 10 patients with DDLPS treated with pembrolizumab in the SARC28 trial, two partial responses were reported, but in subsequent patient expansion, the response rate was only $10 \% .^{12} 13$ The presence of a small subset of patients with DDLPS with tumors responsive to $\mathrm{PD}-1$ inhibition necessitates a better understanding of the tumor microenvironment (TME), with a focus on identification of potential therapeutic targets. Interestingly, these tumors have relatively low expression of PD-1 and generally have few infiltrating $\mathrm{T}$ cells compared with other sarcoma subtypes. ${ }^{14}$ 
To understand the diversity of the DDLPS TME, we undertook the most comprehensive analysis of the DDLPS immune TME performed to date. The analyses correlated patient outcomes with the results of a number of complementary analyses, including multiplex immunohistochemistry (mIHC), tumor escape gene expression analysis, immune cell content quantification, and T-cell receptor (TCR) sequencing. We sought to understand which cell types may be associated with patient outcomes and whether any findings would be suitable targets for novel therapies.

\section{MATERIALS AND METHODS}

\section{Patients and samples}

Eligible patients with DDLPS were those who underwent surgical resection at the University of Washington (UW) between 2002 and 2017, and for whom adequate archival tumor for analysis was identified using Caisis, an opensource web-based cancer data management system for pairing clinical and research information. Tumors were graded according to the French Federation Nationale des centres de Lutte Contre le Cancer (FNCLCC) grading system. ${ }^{15}$ Samples were collected from primary tumors, prior to chemotherapy and/or radiation exposure if possible. Samples from patients undergoing radiation and/or chemotherapy at the time of tissue procurement were excluded.

\section{TCR $\beta$ CDR3 sequencing}

Formalin-fixed paraffin-embedded (FFPE) tissue samples had genomic DNA extracted, and immunosequencing of the CDR3 regions of human TCR $\beta$ chains was performed using the immunoSEQ Assay (Adaptive Biotechnologies, Seattle, Washington, USA) for FFPE to characterize the T-cell fraction and other repertoire metrics, including clonality (online supplemental figure 1 ) ${ }^{16}$ In brief, after amplification of genomic DNA using a bias-controlled, multiplex PCR followed by high-throughput sequencing, sequences were collapsed and filtered in order to identify and quantitate the absolute abundance of each unique TCR $\beta$ CDR3 region for further analysis as previously described. ${ }^{17-19}$

\section{Multiplex immunohistochemistry (mIHC)}

FFPE tissues were stained on a BOND Rx autostainer (Leica Biosystems, Buffalo Grove, Illinois, USA) using the Akoya Opal Multiplex IHC assay (Akoya Biosciences, Menlo Park, California, USA). Additional high stringency washes were performed after the secondary antibody and Opal fluor applications using high-salt TBST $(0.05$ $\mathrm{M}$ Tris, $0.3 \mathrm{M} \mathrm{NaCl}$, and $0.1 \%$ Tween-20, $\mathrm{pH}$ 7.2-7.6). TCT was used as the blocking buffer $(0.05 \mathrm{M}$ Tris, 0.15 $\mathrm{M} \mathrm{NaCl}, 0.25 \%$ casein, $0.1 \%$ Tween $20, \mathrm{pH} 7.6 \pm 0.1$ ). All primary antibodies were incubated for 1 hour at room temperature. OPAL Polymer HRP Mouse plus Rabbit (Akoya Biosciences) was used for all secondary applications (online supplemental figure 2). Slides were mounted with ProLong Gold and cured for 24 hours at room temperature in the dark before image acquisition at $\times 20$ magnification on the Akoya Vectra 3.0 Automated Imaging System. Vectra images were spectrally unmixed using Akoya Phenoptics inForm software and analyzed using the HALO image analysis software Highplex FL module (Indica Labs).

\section{RNA extraction and predictive immune modeling analysis}

Assays were performed in a College of American Pathologists accredited, Clinical Laboratory Improvement Amendments licensed clinical laboratory. Unstained, unmounted FFPE sections from the same FFPE block were processed for RNA extraction using the Prism Extraction Kit (Cofactor Genomics, San Francisco, California, USA). Total RNA was evaluated for quality and quantity using the Bioanalyzer, or TapeStation assay (Agilent, Santa Clara, California, USA), and the Qubit RNA HS or BR Assay (Thermo Fisher, Waltham, Massachusetts, USA). RNA concentration, quantity (in $\mathrm{ng} / \mu \mathrm{L}$ and total ng) and quality (DV200, \% of fragments above $200 \mathrm{nt}$ ) were evaluated to determine library input amount.

Total RNA was processed for library construction by Cofactor Genomics according to the ImmunoPrism Assay standard protocol; $40 \mathrm{ng}$ of RNA was used as input. Libraries were sequenced as single-end 75 base pair reads on a NextSeq500 (Illumina, San Diego, California, USA). An individual ImmunoPrism report, including RNA expression characterization and immune cell quantification, was generated for each sample processed. Immune cell type characterization was defined by RNA models for the ImmunoPrism analysis, ${ }^{20}$ derived from immune cell populations sorted using canonical flow cytometry markers defined by the Human Immunology Project. ${ }^{21}$ Samples were grouped according to clinical outcomes for overall survival (OS), and recurrence-free survival (RFS) and OS and RFS at 3 years was considered a clinical meaningful cut-off point as this has been cited as the median OS for patients with advanced DDLPS. ${ }^{2}$

Gene expression and immune cell quantification were performed using Cofactor's ImmunoPrism platform. ${ }^{22}$ The ImmunoPrism platform is an RNA-based approach to characterizing key immune signals with high sensitivity and specificity. As described previously in Schillebeeckx et al, the platform uses a targeted-capture sequencing and machine learning approach to generate 'health expression models' for accurately identifying percentages of immune cell populations in tumor tissue from RNA expression data. ${ }^{20}$ The immune Health Expression Models (including CD4 + T cells, CD8 + T cells, CD56 + natural killer (NK) cells, CD19 + B cells, CD14 + monocytes, $\mathrm{T}$ regulatory (Tregs) cells, M1 macrophages, and M2 macrophages) used in this study were built using deep sequencing of purified immune cell populations and validated using flow cytometry. The platform also measures a set of immune escape genes including PD-1, PD-L1, CTLA4, OX40, TIM-3, BTLA, ICOS, CD47, IDO1, and ARG1. In a recent publication by James et al, 
exploring immunological signatures in high-grade serous ovarian cancer, the results generated using the ImmunoPrism platform were correlated with the results from an expanded The Cancer Genome Atlas (TCGA) cohort using traditional RNA-seq differential expression analysis methods. ${ }^{23}$

For this study, the potential analytes considered when building the Immunoscore include levels of (1) individual immune cell models (listed above, and reported as a percentage of total cell content of the tissue analyzed), (2) the total immune cell content (listed above, and reported as a percentage of the total cell content of the tissue analyzed), and (3) the expression of immune escape genes (listed above, and reported as transcripts per million, TPM).

\section{Statistical analyses}

Statistical analyses of TCR- $\beta$ sequencing results used Simpson Clonality defined as: Simpsonclonality $=\sqrt{\sum p_{i}^{2}}$, and was calculated on productive rearrangements, where $p_{i}$ is the proportional abundance of rearrangement $i$ and $N$ is the total number of rearrangements. Clonality values range from 0 to 1 and describe the shape of the frequency distribution. Clonality values approaching 0 indicate a very even distribution of frequencies, whereas values approaching 1 indicate an increasingly asymmetric distribution in which a few clones are present at high frequencies. Statistical analysis was performed in R V.3.2. The fraction of T cells in FFPE tissue samples was calculated by normalizing TCR- $\beta$ template counts to the total amount of DNA usable for TCR sequencing, where the amount of usable DNA was determined by PCR amplification and sequencing of several housekeeping genes that are expected to be present in all nucleated cells. Additionally, Pearson correlation coefficient analysis was performed to evaluate immune markers used for mIHC.

Descriptive statistics were used to evaluate the mean differences between recurrence groups including t-tests for continuous data and $\chi^{2}$ tests for categorical data. Creation of the prognostic multi-analyte classifier was generalized from methods advocated by Harrell et $a l^{24}$ and Moons et $a l^{25}$ and implemented to describe the optimal relationship between immune analytes. Statistical methods included exploratory data analyses, variable selection, qualitative cut-off development, and model performance evaluation. We evaluated between-analyte multicollinearity using clustering, recursive partitioning, and principal component analysis. Selection of important predictors and creation of a classification score were performed in two stages using the lasso with an elastic net algorithm. ${ }^{26}$

Elastic-net regularization was used to perform variable selection through a shrinkage parameter. Often there is no form solution for the shrinkage parameter; however, it can be estimated using an optimization algorithm ${ }^{27}$ that minimizes the centered and standardized (to remove effects of scaling) functions. We used selected predictors to create a score viewed as a weighted sum, describing
Table 1 Demographics and characteristics of patients with DDLPS

\begin{tabular}{|c|c|}
\hline Characteristics & $n(\%)(N=61)$ \\
\hline \multicolumn{2}{|l|}{ Age (years) } \\
\hline $0-40$ & $0(0)$ \\
\hline $41-50$ & 2 (3) \\
\hline $51-60$ & $11(18)$ \\
\hline $61-70$ & $17(28)$ \\
\hline $71-80$ & $20(33)$ \\
\hline $81-90$ & $11(18)$ \\
\hline \multicolumn{2}{|l|}{ Gender } \\
\hline Female & $25(41)$ \\
\hline Male & $36(59)$ \\
\hline \multicolumn{2}{|l|}{ Sarcoma type } \\
\hline DDLPS & $61(100)$ \\
\hline \multicolumn{2}{|l|}{ Primary tumor } \\
\hline Yes & $47(77)$ \\
\hline No & $14(23)$ \\
\hline \multicolumn{2}{|l|}{ Treatment prior to surgery } \\
\hline None & $47(77)$ \\
\hline RT & $7(11)$ \\
\hline Chemotherapy & $3(5)$ \\
\hline Chemotherapy and RT & $4(7)$ \\
\hline \multicolumn{2}{|l|}{ FNCLCC tumor grade } \\
\hline 1 & $16(26)$ \\
\hline 2 & $24(39)$ \\
\hline 3 & $21(34)$ \\
\hline \multicolumn{2}{|l|}{ Tumor size, cm } \\
\hline Mean (SD) & $21.2(12)$ \\
\hline \multicolumn{2}{|l|}{ Multiple tumors (\%) } \\
\hline Yes & $7(11)$ \\
\hline No & $54(89)$ \\
\hline \multicolumn{2}{|l|}{ Vital status } \\
\hline Alive & $29(48)$ \\
\hline Deceased & $32(52)$ \\
\hline \multicolumn{2}{|l|}{ Recurrence } \\
\hline Yes & $37(61)$ \\
\hline No & $24(39)$ \\
\hline \multicolumn{2}{|c|}{ Recurrence-free survival, months } \\
\hline Mean (SD) & $41.5(40.1)$ \\
\hline Range & $5.1-196.9$ \\
\hline \multicolumn{2}{|l|}{ Overall survival, months } \\
\hline Mean (SD) & $74.8(62.8)$ \\
\hline Range & $3.3-236.4$ \\
\hline
\end{tabular}

DDLPS, dedifferentiated liposarcoma; FNCLCC, French Federation Nationale des centres de Lutte Contre le Cancer; RT, Radiation Therapy.

both the direction of the association (those that are associated with recurrence vs those that are more associated with non-recurrence) and the relative strength (eg, large weights contribute more to the score).

Performance characteristics of the prognostic classifier include sensitivity, specificity, and area under the receiver 
Table 2 Impact of clinical variables and T-cell repertoire metrics on RFS at 3years

\begin{tabular}{|c|c|c|c|}
\hline & $\begin{array}{l}\text { No recurrence } \\
(n=20)\end{array}$ & $\begin{array}{l}\text { Recurrence } \\
(\mathrm{n}=29)\end{array}$ & $P$ value \\
\hline \multicolumn{4}{|l|}{ Sex } \\
\hline Female & $9(45.0 \%)$ & $9(31.0 \%)$ & 0.995 \\
\hline Male & $11(55.0 \%)$ & 20 (69.0\%) & \\
\hline \multicolumn{4}{|l|}{ Age } \\
\hline Mean (SD) & $73.4(12.1)$ & $68.4(7.91)$ & 0.632 \\
\hline Median (min, max) & $75.0(50.0,89.0)$ & $69.0(54.0,83.0)$ & \\
\hline \multicolumn{4}{|l|}{ Grade 2} \\
\hline High & $5(25.0 \%)$ & $10(34.5 \%)$ & NA \\
\hline Low/intermediate & $15(75.0 \%)$ & $19(65.5 \%)$ & \\
\hline$P$ value & $0(0 \%)$ & $0(0 \%)$ & \\
\hline \multicolumn{4}{|l|}{ T cell infiltrates } \\
\hline High & 7 (35.0\%) & $4(13.8 \%)$ & 0.13 \\
\hline Low & $2(10.0 \%)$ & $8(27.6 \%)$ & \\
\hline Moderate & $11(55.0 \%)$ & 17 (58.6\%) & \\
\hline \multicolumn{4}{|l|}{ Clonality } \\
\hline High & $9(45.0 \%)$ & 12 (41.4\%) & 0.194 \\
\hline Moderate/low & $11(55.0 \%)$ & 17 (58.6\%) & \\
\hline \multicolumn{4}{|l|}{ T cells total } \\
\hline Mean (SD) & $0.165(0.146)$ & $0.170(0.134)$ & 0.289 \\
\hline Median (min, max) & $0.105(0.0100,0.620)$ & $0.140(0.0200,0.670)$ & \\
\hline \multicolumn{4}{|l|}{ Overall survival } \\
\hline Mean (SD) & $4.68(4.60)$ & $3.34(3.18)$ & 0.98 \\
\hline Median (min, max) & $2.60(0.300,17.4)$ & $2.00(0.400,13.8)$ & \\
\hline \multicolumn{4}{|c|}{ Total immune content } \\
\hline Mean (SD) & $0.365(0.225)$ & $0.372(0.218)$ & 0.678 \\
\hline Median (min, max) & $0.305(0.100,0.960)$ & $0.320(0.0700,0.900)$ & \\
\hline
\end{tabular}

A t-test was used for continuous data, and a $\chi^{2}$ test was used for categorical data to analyze clinical characteristics and immunosequencing data in relation to RFS at 3 years.

RFS, recurrence-free survival.

operating characteristic curve (AUC). Overfitting was assessed using bootstrap cross-validation. All analyses used reproducible methods via the $\mathrm{R}$ statistical package ( www.rproject.org) and R-markdown (http://rmarkdown. rstudio.com/). These methods allow for all analyses, associated tables, listings, and figures to be reconstructed in their entirety in a single document. Each step in the modeling process, including quality control (QC) and intermediate data output, was programmed in the smallest executable processes ('chunks' in markdown). All packages (including the base R package) were locally sourced to ensure long-term compatibility.

\section{RESULTS}

\section{Clinical demographics}

In total, 61 patients were included in this study (table 1). The mean age was 71 years (range 48-89). All patients had DDLPS, and 47 (77\%) were primary tumors. Fortyseven $(77 \%)$ patients had no treatment prior to tissue collection, only three $(5 \%)$ had preoperative radiation within 6 months prior to tissue collection, and only three $(5 \%)$ received chemotherapy within 12 months of tissue collection. The most common tumor grade by FNCLCC classification was intermediate (ie, grade 2), $24(39 \%)$. The mean tumor size was $21.2 \mathrm{~cm}( \pm 12)$, and seven $(11 \%)$ patients had multiple tumors. At the time of this analysis, $29(48 \%)$ patients were alive. Recurrence was seen in 37 (61\%), with a mean RFS of 41.5 months (range 5.1-196.9) and a mean OS of 74.8 months (range 3.3-236.4). The median follow-up time was 34.4 months (range 1.4-236.4).

\section{Quantification of escape genes in DDLPS}

Expression of common cancer escape genes was assessed to understand whether DDLPS might hinder an 
A)

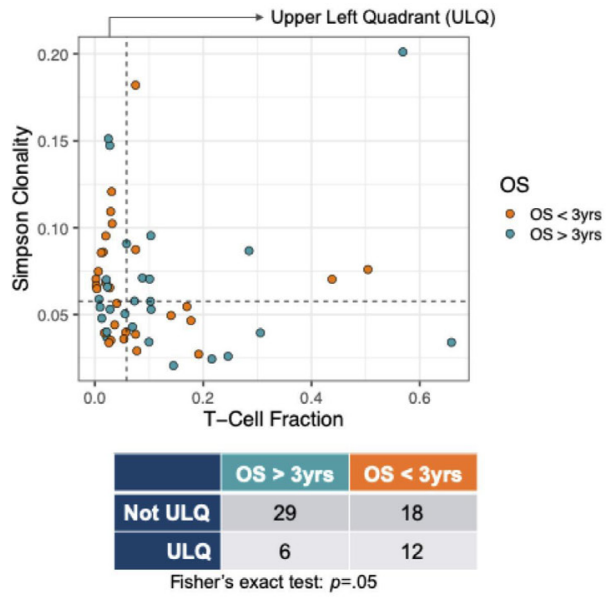

B)
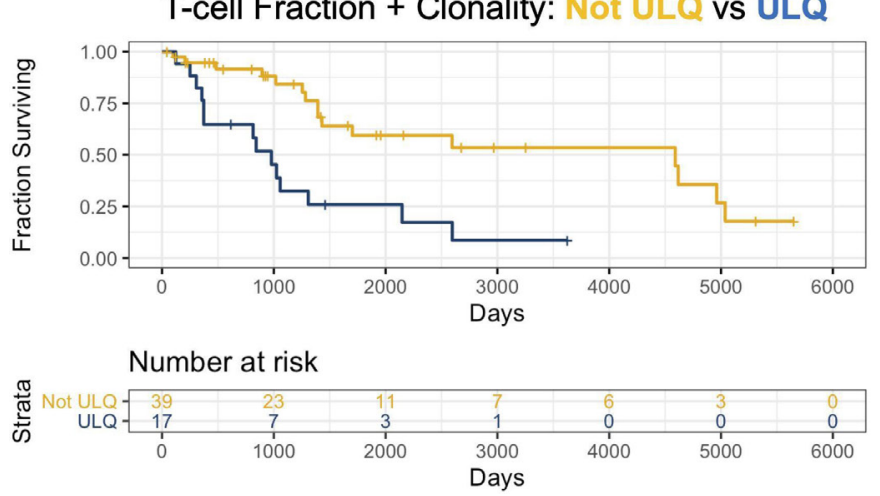

Figure 1 T-cell fraction and clonality in relation to 3-year overall survival (OS). (A) Combined T-cell repertoire clonality and T-cell fraction subdivided into quadrants based on mean values for each metric. Relative 3-year OS is reported for each quadrant. (B) Cox regression for tumor T-cell fraction and repertoire clonality in relation to OS in patients with dedifferentiated liposarcoma. HR $p<0.001$. Likelihood ratio test $p<0.001$. ULQ, upper left quadrant.

effective immune response leading to early recurrence and inferior OS. Forty-nine tumors had high-quality RNA extracted for sequencing and immune modeling using ImmunoPrism. Ten genes were prospectively selected for our initial analysis: PD-1, PD-L1, CTLA-4, OX40, TIM3, BTLA, ICOS, CD47, IDO1, and ARG1 (online supplemental figure 3). $P D-1$ was more highly expressed in patients with greater than 3-year OS (cut-off=966.02 TPM, $\mathrm{p}=0.003)$. Other markers more highly expressed in 3-year survivors include ICOS (cut-off $=415.50$ TPM, $\mathrm{p}=0.006$ ), BTLA (cut-off=496.77TPM, p=0.03), and CTLA-4 (cutoff $=1283.30$ TPM, $\mathrm{p}=0.02)$.

\section{T-cell fraction, clonality and clinical outcomes}

DDLPS samples from 61 patients were available to characterize the repertoire of T cells using the immunoSEQAssay. The mean number of $\mathrm{T}$ cells in samples was 6303 (range 49-117,420) (online supplemental figure 4). The mean number of T-cell clones was 4001 (range 49-39,581), and the mean clonality was $0.066(0.021-0.201)$. These data suggest that most patients had a diverse, but not clonal T-cell repertoire. The mean clone frequency among the samples was $0.037(0.006-0.196)$. The mean number of total nucleated cells per FFPE tissue sample was 67,188 (1049-268,036), and the mean total T-cell fraction was $0.104(0.002-0.659)$.

Multivariable analysis of clinical characteristics and immunosequencing data was performed in relation to 3-year RFS (table 2). Levels of T-cell infiltration $(\mathrm{p}<0.001)$ and clonality $(\mathrm{p}<0.001)$ above the median were significantly associated with superior 3-year RFS. Neither sex nor age was statistically significant independent variables $(\mathrm{p}=0.76$ and $\mathrm{p}=0.79)$, and tumor grade was not a statistically significant independent factor for RFS at 3 years $(\mathrm{p}=0.38)$.

The relationships between T-cell fraction, clonality and OS were analyzed using a Cox regression model. We discovered that higher T-cell fraction had a trend toward improved survival (HR p=0.07, Likelihood Ratio Test (LRT) p=0.04) (online supplemental figure 5). Additionally, the presence of multiple tumors (HR $\mathrm{p}=0.02$, LRT $\mathrm{p}=0.03$ ) and older patient age were associated with inferior OS (HR p=0.02, LRT $\mathrm{p}=0.02)$. However, neither tumor grade nor T-cell clonality $(\mathrm{HR} p=0.72$, LRT $\mathrm{p}=0.71)$ was independently associated with OS.

In order to look more closely at the relationship between the T-cell repertoire metrics as determined by immunosequencing and OS, both T-cell fraction and clonality were subdivided into quadrants based on mean values for each metric among all patients (figure 1). Although neither T-cell fraction nor clonality was independently associated with OS (online supplemental figures 5 and 6), patients with both a low T-cell fraction and a high T-cell clonality (figure 1) had lower OS $(p=0.05)$. Patients with these characteristics related to their $\mathrm{T}$-cell repertoire represented nearly half of all patients who died within 3 years from diagnosis.

\section{Quantification of tumor-infiltrating immune cells in LPS using ImmunoPrism}

The ImmunoPrism platform used RNA sequencing data to evaluate the cellular immune profile. ${ }^{20}$ Tumor-infiltrating immune cells selected for analysis included CD4 + T cells, CD8 + T cells, CD19 + B cells, CD14 + monocytes, CD56 + NK cells, M1 macrophages, M2 macrophages, and Tregs. The overall mean percent of immune cells per DDLPS sample was $36.9 \%( \pm 21.8)$ of the total cell population, and the most prevalent infiltrating immune cells were CD14+ monocytes, with a mean of $7.6 \%( \pm 7.6)$ of the total cell population. The most prevalent infiltrating lymphocytes were CD8 + Tcells, with a mean of $7.1 \%( \pm 10.3)$ of the total cell population (online supplemental figure 7). M1 macrophages only constituted, on average, $0.1 \%$ of the total cell population and M2 macrophages were nearly 25 times more prevalent, with a mean overall percent of $2.3 \%( \pm 1.6)$. Additionally, Tregs represented $4.2 \%$ of all cells. 


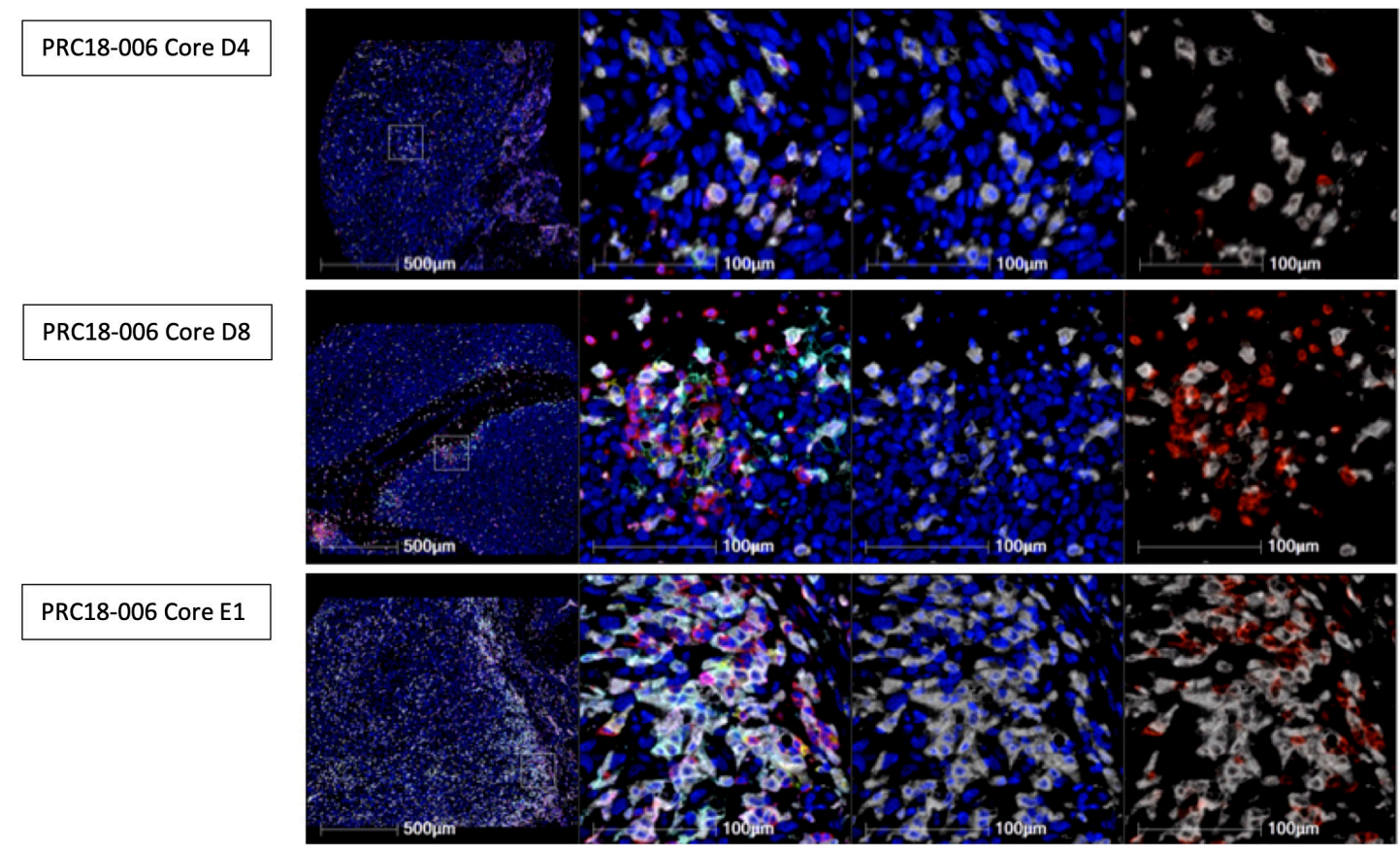

Figure 2 Dedifferentiated liposarcoma multiplex immunohistochemistry of representative patients. Rows 1, 2, and 3 (left to right): Core at $\times 5$ magnification with all colors, $\times 40$ with all colors, $\times 40$ with CD68/CD163 and DAPI, $\times 40$ with CD68/CD163 and CD3. CD11c, green; CD16, light blue; HLA-DR, yellow; CD14, magenta; CD3, red; CD68/CD163, white; DAPI, dark blue.

The overall number of immune cells was not associated with OS. Patients with greater than 3-year OS had lower levels of M2 macrophages (3.3\% vs $1.96 \%, \mathrm{p}=0.05)$ and CD14 + monocytes $(10.72 \%$ vs $6.29 \%, p=0.13)$. Patients who had a greater than 3-year RFS had a higher number of $\mathrm{CD} 4$ + Tcells (cut-off $=5.49 \%, \mathrm{p}=0.05$ ). Interestingly, in patients who had an intermediate grade tumor, those with higher populations of CD19 + B cells (cut-off $=2.22 \%$, $\mathrm{p}=0.02$ ) or CD4 + Tcells (cut-off $=5.49 \%, \mathrm{p}=0.03$ ) were more likely to remain recurrence free at 3 years.

To better characterize and confirm cell populations identified with ImmunoPrism, mIHC was performed using established methods ${ }^{28}$ (online supplemental figure 8) on a separate, clinically similar cohort containing 22 DDLPS patient samples. Staining routinely demonstrated a significant proportion of CD68+/CD163 + cells (figure 2), and pairwise correlation was performed to demonstrate consistency with previous findings using the ImmunoPrism assay with CD14 correlated to PD-1/PD-L1 and markers for macrophages, which is consistent with the Immunoscore model and exploratory data analyses (figure 3). CD14+, CD11c+, and HLA-DR + cells were also appreciated, illustrating the prevalence of tumorassociated macrophages (TAMs) in WD/DD LPS.

\section{Relationships among infiltrating immune cells and escape genes}

Given the statistically significant association (table 2) between 3-year RFS and either T-cell infiltrates $(p<0.001)$ or clonality $(\mathrm{p}<0.001)$, genetic and cellular relationships were explored to identify more granular predictors of outcome. We constructed principal component biplots for both gene expression and immune cell types (figure 4A and B). These plots were performed after standardizing and centering genes and immune cells. Despite overlap in confidence ellipses, likely related to limited sample size, multiple relationships were observed. Notably, inverse relationships between CD8 + Tcells, M1 macrophages, and M2 macrophages were appreciated, along with inverse relationships between CD19 + B cells, CD14 + monocytes and Tregs (figure 4A). With this analysis, typically only one to two elements would cluster with such high similarity. Interestingly, ARG1 (figure 3B) and CD8 T cells (figure 4A) load strongest in patients with poorer outcomes $(\leq 3$-year RFS), while CD19 + B cells loaded strongest in the patients with better outcomes (>3-year RFS) (figure 4A).

To further examine these relationships between escape genes, immune cells, and the combination of both, we created hierarchical clustering using the Hoeffding D statistic as a pairwise similarity measure (figure 4C-E). programmed cell death protein 1 (PD-1) and cytotoxic T-lymphocyte associated protein 4 (CTLA-4) were highly similar (Hoeffding D of 0.4 ), while B and T lymphocyte attenuator (BTLA) and inducible costimulator (ICOS) also clustered when relaxing the Hoeffding $\mathrm{D}$ similarity to 0.3 (figure 4C). Although the similarity measure between immune cells is lower, a clear cluster emerged between CD14 + monocytes and M2 macrophages (Hoeffding $\mathrm{D}$ of 0.12) (figure 4D). Interestingly, CD8 + Tcells and TIM3 showed high similarity (figure 4E). Additionally, M1 macrophages, CD19 + B cells and Tregs clustered with PD-1, CTLA-4, PD-L1, and BTLA genes; while the CD56 + NK cells, CD4 + T cells, CD14 + monocytes and M2 macrophages clustered with indoleamine 2,3-dioxygenase 1 (IDO1), arginase 1 (ARG1), and OX40 (figure 4E). 


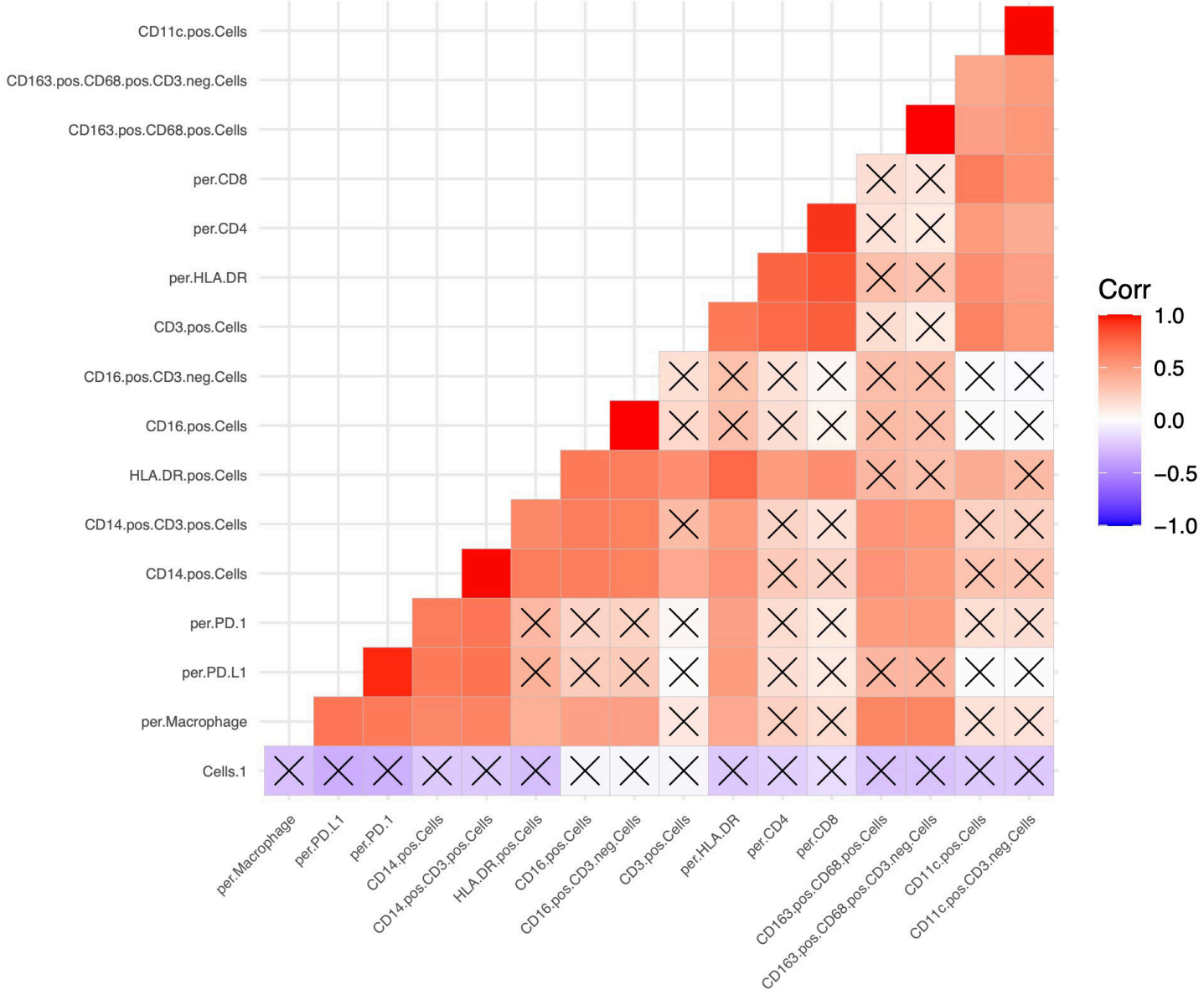

Figure 3 Pairwise correlation for multiplex immunohistochemistry (mIHC). The heat map demonstrates pairwise correlation between $\mathrm{mlHC}$ markers where red represents a strong positive correlation, and blue represents a strong negative correlation. All boxes are statistically significant with a $\mathrm{p} \leq 0.05$, except for boxes marked with an ' $\mathrm{X}$ '.

Additionally, a recursive partitioning was performed for genes, cell types, and a combination of both, with the aim of developing a predictive model for OS in patients with LPS (online supplemental figure 9). From this recursive partitioning, CTLA-4 was the decisive marker that subsequently split into PD-L1 and ICOS. High expression of CTLA-4 (cut-off $>3441$ TPM) alone predicted $20 \%$ of all patients that survived greater than 3 years. Furthermore, $50 \%$ of patients with $\leq 3$-year OS are captured by partitioning for CTLA-4, PD-L1, and ICOS, while over $40 \%$ of patients with $>3$-year OS are also captured by these same three genes (online supplemental figure 9).

\section{Creation of DDLPS Immunoscore}

Three immune components were selected from the elastic net algorithm to create an immune score for DDLPS in predicting outcomes. This included CD4 + Tcells, CD14 + monocytes, and M2 macrophages. A linear score was developed, in which weights greater than zero are more highly expressed in patients with >3-year RFS; weights less than zero are associated with $\leq 3$-year RFS (figure 5 ). The gene expression score alone demonstrated little statistical predictive capacity (AUC $=0.550$, not shown). However, when immune markers (ie, cell types) were combined with genes, the Immunoscore for the AUC improved from 0.752 to 0.789 , indicating improved statistical prediction. Importantly, when tumor site was included in analysis (ie, retroperitoneal, limb, etc), it had no impact on the Immunoscore (online supplemental figure 10). There was little evidence of overfitting in either model, with $1 \%$ in the immune score model, and $6 \%$ for the immune score and gene scores combined. Overfitting was mitigated by dimension reduction using the linear combination of immune analytes and gene expression as scores with appropriate shrinkage estimators applied rather than individual predictors.

\section{DISCUSSION/CONCLUSION}

In this study, we retrospectively studied FFPE tumor specimens from 61 patients with LPS and analyzed gene expression and immunosequencing data with regard to clinical outcomes. Our data suggest that LPS represents diseases with varying immunophenotypes that were associated with different clinical outcomes. Consistent with prior findings, ${ }^{29}$ we showed that LPS generally has low levels of T-cell clonality. When combined with high T-cell fraction, patients had significantly worse outcomes. This may indicate there exists a patient subset with highly 


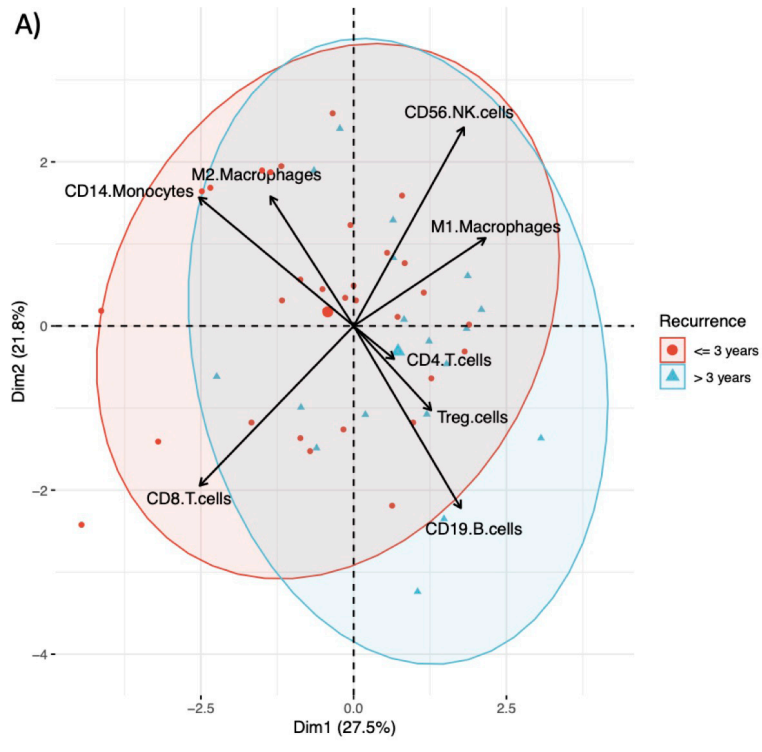

C)

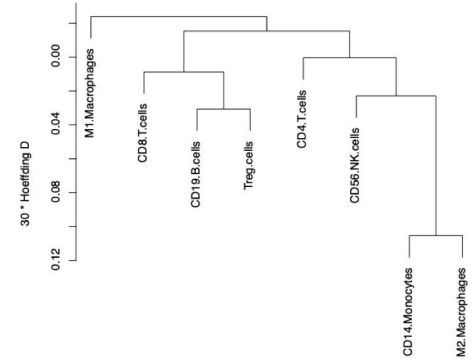

B)

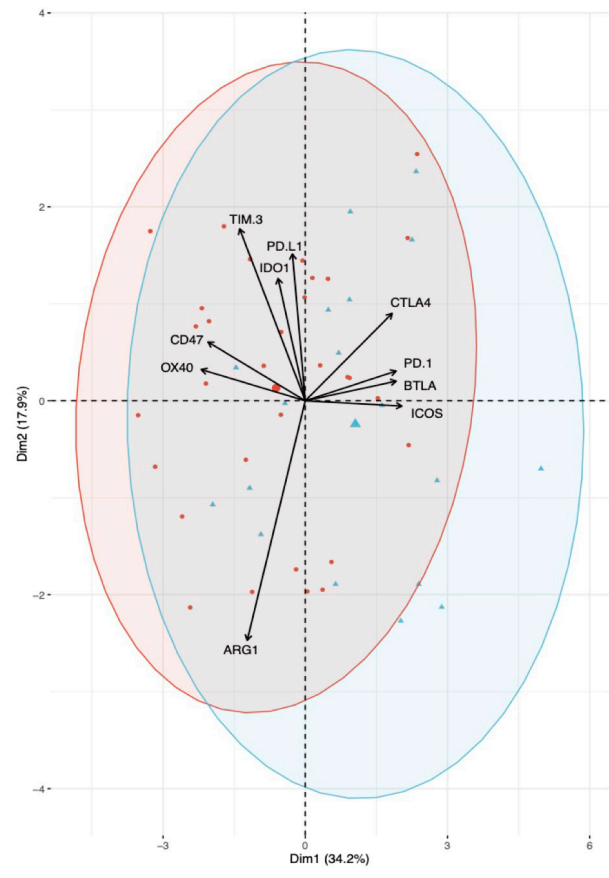

D)

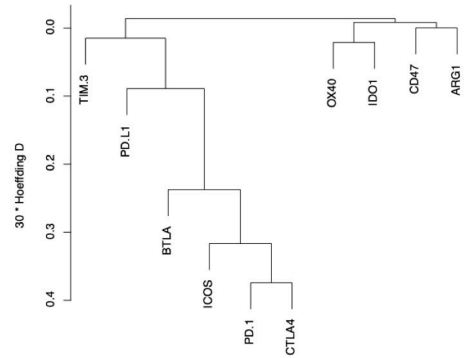

E)

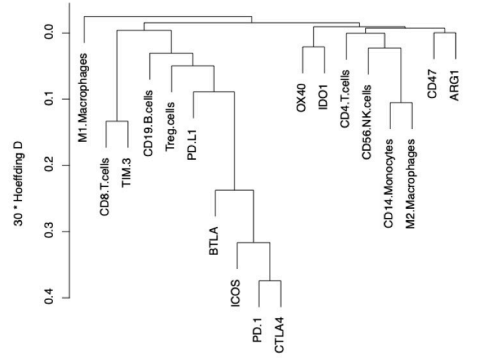

Figure 4 Hierarchical clustering using the Hoeffding D statistic as a pairwise distance measure and biplots based on 3-year overall survival (OS). Biplots based on patient 3-year OS using immune cells (A) and escape genes (B). Example of inverse relationships include between CD8+ Tcells, M1 macrophage and M2 macrophages, and additionally between CD19+ B cells, CD14+ monocytes and regulatory T-cells. Example of multicolinear relationship between CD4+ Tcells and CD19+ B cells. Hierarchical clustering with immune cells (C), escape genes (D), and combined (E). Tregs, regulatory T cells. ARG1, arginase 1; BTLA, B and T lymphocyte attenuator; CD, cluster of differentiation; CTLA-4, cytotoxic T-lymphocyte associated protein 4; ICOS, inducible costimulator; PD-1, programmed cell death protein 1; TIM3, T cell immunoglobulin and mucin-domain containing 3.

inflammatory tumors lacking strong, specific immunogenic targets. Further efforts to study the DDLPS TME should distinguish these patients from patients with DDLPS with tumors that have a quiet TME, with few T cells, and those who have a high clonality and presumably antigen-specific inflammatory response.

As has been seen in prior studies, higher PD-1/PD-L1 correlated with patient outcomes. ${ }^{30}$ PD-1 targeted therapies have yielded relatively low objective response rates $(10 \%-20 \%)$ in DDLPS but in those patients with responses, they have tended to be durable. ${ }^{12}$ Patients with fewer monocytic cells (particularly TAMs), and those with higher CD4 + T cells, were also identified as having better clinical outcomes. In fact, CD4 + T cells were most strongly associated with better outcomes in this analysis. This finding is consistent with a growing body of literature. Tseng et al observed that the majority of infiltrating lymphocytes in RLPS are CD4 + and are frequently juxtaposed to DC-LAMP positive mature dendritic cells. ${ }^{30}$ Furthermore, Petitprez et al characterized tumors based on sarcoma immune classes, and of those classes, they discovered better outcomes were more likely in patients with high expression of PD-1, CTLA4, and TIM3 $3{ }^{31}$ These findings are similar to ours, although TIM3 did not reach statistical significance in our cohort.

Perhaps the most interesting cell population identified from expression profiling was the significant prevalence of M2 macrophages. This prevalent cell type likely contributes to an immunosuppressive TME. Therefore, therapies that have anti-macrophage activity could be promising including those using standard cytotoxic chemotherapies. ${ }^{32}$ Trabectedin has shown direct cytotoxic effects on TAMs,${ }^{33-35}$ and future studies in DDLPS could consider this treatment strategy, possibly in conjunction with 


\begin{tabular}{|c|c|c|}
\hline \multicolumn{2}{|l|}{ Marker } & Weight \\
\hline \multicolumn{2}{|l|}{ CD4.T.cells } & 6.64 \\
\hline \multicolumn{2}{|l|}{ CD14.Monocytes } & -2.83 \\
\hline \multicolumn{2}{|l|}{ M2.Macrophages } & -3.98 \\
\hline Model & P-Value & $\begin{array}{c}\text { AUC } \\
\text { (adjusted) }\end{array}$ \\
\hline $\begin{array}{l}\text { Immune Marker } \\
\text { Score }\end{array}$ & 0.0014 & $0.752(0.745)$ \\
\hline $\begin{array}{l}\text { Immune Marker + } \\
\text { Genes }\end{array}$ & 0.0116 & $0.771(0.696)$ \\
\hline $\begin{array}{l}\text { Immune Marker + } \\
\text { Genes + Grade }\end{array}$ & 0.0122 & $0.778(0.683)$ \\
\hline
\end{tabular}

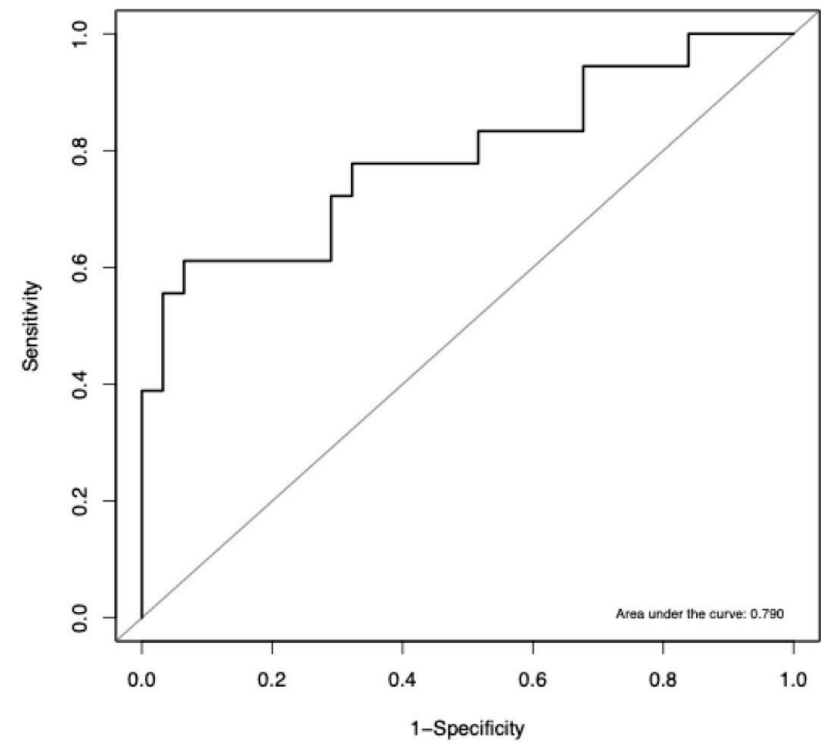

Figure 5 Three immune components were selected from the elastic net algorithm to create an immune score (top) for predicting patient outcomes in which weights greater than zero are more highly expressed in patients with greater than 3-year recurrence-free survival. The adjusted area under the receiver operating characteristic curve (AUC) (bottom) used to measure strength of predictions increased when immune markers were combined with genes demonstrating improved statistical prediction.

checkpoint inhibition given what we have demonstrated here.

Several precautionary comments are important to the interpretation of the data presented here. Although this is one of the largest cohorts of patients with DDLPS, the overall sample size is a study limitation. Furthermore, despite our best efforts, five patients received treatment within 1 year of sample collection, which could affect the TME and serve as a confounder. In future efforts we hope to validate our prognostic score in an independent cohort.

Despite these precautionary comments, the results of our exploratory hypothesis-generating study indicate substantial, and clinically important heterogeneity in the immunophenotype of patients with DDLPS. These differences may be exploited to predict patient outcomes and identify immunologically tailored treatment regimens. Patients with higher levels of PD-1 and CTLA-4 expression are more likely to recur later than 3 years versus those with lower expression levels. The TME appears to play an integral role in tumor suppression as evidenced by increased time to tumor recurrence with increased PD-1 and CTLA-4 expression. Furthermore, lower levels of immunosuppressive CD14 + monocytes and M2 macrophages are associated with better patient outcomes. Similar to what others have shown, ${ }^{31}$ we found that CD19 + B cells were associated with better outcomes, although CD4 + Tcells represent the single most powerful positive predictor. Future studies in DDLPS in the advanced setting may consider combining checkpoint inhibition with TAM depleting strategies, while incorporating immunosequencing, as well as gene expression profiling techniques, such as ImmunoPrism, to generate comprehensive immune profiles in order to better identify potential responders.
In addition, it may be possible to exploit assessment of CTLA-4 and PD-1 expressions to provide more reliable patient prognostication.

\section{Author affiliations \\ ${ }^{1}$ National Cancer Institute, National Institutes of Health, Bethesda, Maryland, USA ${ }^{2}$ Clinical Research Division, Fred Hutchinson Cancer Research Center, Seattle, Washington, USA \\ ${ }^{3}$ Cofactor Genomics, San Francisco, California, USA \\ ${ }^{4}$ BI05 Institute, University of Arizona, Tucson, Arizona, USA \\ ${ }^{5}$ Adaptive Biotechnologies Corp, Seattle, Washington, USA \\ ${ }^{6}$ Bristol Myers Squibb, Seattle, Washington, USA \\ ${ }^{7}$ UW Medicine Cancer Vaccine Institute, University of Washington, Seattle, Washington, USA \\ ${ }^{8}$ Division of Oncology, Northwestern University Department of Medicine, Chicago, Illinois, USA \\ ${ }^{9}$ Department of Surgery, University of Washington, Seattle, Washington, USA \\ ${ }^{10}$ Department of Laboratory Medicine and Pathology, University of Washington, Seattle, Washington, USA \\ ${ }^{11}$ Division of Oncology, University of Washington, Seattle, Washington, USA \\ ${ }^{12}$ Pathology, University of Washington Medical Center, Seattle, Washington, USA \\ ${ }^{13}$ Sensei Bio, Boston, Massachusetts, USA \\ ${ }^{14}$ Sarcoma, Royal Marsden Hospital NHS Trust, London, UK \\ ${ }^{15}$ Seattle Cancer Care Alliance, Seattle, Washington, USA \\ ${ }^{16}$ Division of Oncology, Northwestern University, Chicago, Illinois, USA}

Twitter Brett A Schroeder Brett A Schroeder @oncdocbrett, Natalie A LaFranzo Natalie A LaFranzo @CheerChemist, Bonnie J LaFleur Bonnie A LaFleur @bonnie_ lafleur, Rachel M Gittelman Rachel M Gittelman @RachelGittelman, Marissa Vignali Marissa Vignali @mgvignali, Laura Riolobos Laura Riolobos @riolobos_laura, Teresa S Kim @safffffire, Michael J Wagner Michael J Wagner @wagsmd, Robin L Jones Robin L Jones @RobinL_Jones and Seth M Pollack @immunosarc

Contributors BAS compiled and analyzed clinical data, prepared and edited manuscript. NAL analyzed data, prepared and edited manuscript. BJL performed technical data analysis and edited manuscript. RMG edited manuscript and analyzed data. MV, SZ, KCF, LRS, BCS, MJW, TSK, JGM, EC, JSC, and RHP edited manuscript. JR performed technical data analysis. KSS performed experiments. RLJ and LDC prepared and edited manuscript. SMP provided leadership, prepared manuscript, and obtained funding. 
Funding Funding for this study was provided by CA180380 and the Sarcoma Foundation of America. SMP was also supported by R01CA244872, CA180380, a grant from the V Foundation, and the Seattle Translational Tumor Research (STTR).

Competing interests BAS, SZ, LRS, BCS, KSS, JGM, EC, JSC, and RHP declare no potential conflicts of interest. SMP reported research funding from Merck during the conduct of the study; research funding from EMD Serono, Incyte, Presage, Janssen, OncoSec, and Juno and consulting, honoraria, and advisory activity with GlaxoSmithKline, Eli Lilly and Company, Seattle Genetics, Bayer, Tempus, Daiichi Sankyo, and Blueprint Medicine, outside the submitted work. NAL and KCF are employed by Cofactor Genomics, Inc, the company that developed and produces the ImmunoPrism ${ }^{\circledR}$ reagent kit and informatics tools used in this article. BJL is a paid consultant for Cofactor Genomics, Inc. RMG and MV have a financial interest in Adaptive Biotechnologies. JR has equity in Adaptive Biotechnologies, equity and employment with Bristol Myers Squibb. MJW reported research funding from Athenex, Deciphera, Incyte, Tempus, Adaptimmune, and GlaxoSmithKline and consulting, honoraria, and advisory activity with Tempus, Deciphera, and Adaptimmune, outside the submitted work. RLJ reported grants from Merck during the conduct of the study; research support from Merck Sharp \& Dohme and GlaxoSmithKline and consultation fees from Adaptimmune, Athenex, Blueprint Medicine, Clinigen, Eisai, Epizyme, Daiichi Sankyo, Deciphera, Immune Design, Eli Lilly and Company, Merck, Pharma Mar, and UpToDate, outside the submitted work in addition, RLJ had a patent to biomarker, issued. LDC receives research funding, paid to institution, from Eli Lilly, AADi, BluePrint Medicine, Iterion, Gradalis, Philogen, Advenchen Laboratories, and CBA Pharma. LDC institution has received funding from Eli Lilly for conduct of clinical trials. LDC has received honoraria or has served on advisory boards for BluePrint Medicines and Regeneron.

Patient consent for publication Not required.

Ethics approval De-identified patient data were used for the analysis under a minimal-risk protocol approved by the Institutional Review Board (IRB) of the Fred Hutchinson Cancer Research Center (FHCRC).

Provenance and peer review Not commissioned; externally peer reviewed.

Data availability statement Data are available on reasonable request. All data relevant to the study are included in the article or uploaded as supplementary information.

Supplemental material This content has been supplied by the author(s). It has not been vetted by BMJ Publishing Group Limited (BMJ) and may not have been peer-reviewed. Any opinions or recommendations discussed are solely those of the author(s) and are not endorsed by BMJ. BMJ disclaims all liability and responsibility arising from any reliance placed on the content. Where the content includes any translated material, BMJ does not warrant the accuracy and reliability of the translations (including but not limited to local regulations, clinical guidelines, terminology, drug names and drug dosages), and is not responsible for any error and/or omissions arising from translation and adaptation or otherwise.

Open access This is an open access article distributed in accordance with the Creative Commons Attribution Non Commercial (CC BY-NC 4.0) license, which permits others to distribute, remix, adapt, build upon this work non-commercially, and license their derivative works on different terms, provided the original work is properly cited, appropriate credit is given, any changes made indicated, and the use is non-commercial. See http://creativecommons.org/licenses/by-nc/4.0/.

\section{ORCID iDs}

Brett A Schroeder http://orcid.org/0000-0001-6019-2022

Shihong Zhang http://orcid.org/0000-0003-1373-2668

Teresa S Kim http://orcid.org/0000-0002-6040-7748

Michael J Wagner http://orcid.org/0000-0002-0753-9282

Robert H Pierce http://orcid.org/0000-0002-2677-144X

Seth M Pollack http://orcid.org/0000-0002-2466-0607

\section{REFERENCES}

1 Bock S, Hoffmann DG, Jiang Y, et al. Increasing incidence of liposarcoma: a population-based study of national surveillance databases, 2001-2016. Int J Environ Res Public Health 2020;17:2710.

2 Tirumani SH, Tirumani H, Jagannathan JP, et al. Metastasis in dedifferentiated liposarcoma: predictors and outcome in 148 patients. Eur J Surg Oncol 2015;41:899-904.

3 Demetri GD, von Mehren M, Jones RL, et al. Efficacy and safety of trabectedin or dacarbazine for metastatic liposarcoma or leiomyosarcoma after failure of conventional chemotherapy: results of a phase III randomized multicenter clinical trial. JCO 2016;34:786-93.

4 Schöffski P, Chawla S, Maki RG, et al. Eribulin versus dacarbazine in previously treated patients with advanced liposarcoma or leiomyosarcoma: a randomised, open-label, multicentre, phase 3 trial. Lancet 2016;387:1629-37.

5 Fletcher CDM. The evolving classification of soft tissue tumours - an update based on the new 2013 WHO classification. Histopathology 2014:64:2-11.

6 Singer S, Socci ND, Ambrosini G, et al. Gene expression profiling of liposarcoma identifies distinct biological types/subtypes and potential therapeutic targets in well-differentiated and dedifferentiated liposarcoma. Cancer Res 2007;67:6626-36.

7 Thway K, Jones RL, Noujaim J, et al. Dedifferentiated liposarcoma: updates on morphology, genetics, and therapeutic strategies. Adv Anat Pathol 2016;23:30-40.

8 Cissé MY, Pyrdziak S, Firmin N, et al. Targeting MDM2-dependent serine metabolism as a therapeutic strategy for liposarcoma. Sci Trans/ Med 2020;12:eaay2163.

9 Dickson MA, Tap WD, Keohan ML, et al. Phase II Trial of the CDK4 Inhibitor PD0332991 in Patients With Advanced CDK4 -Amplified Well-Differentiated or Dedifferentiated Liposarcoma. JCO 2013;31:2024-8.

10 Dickson MA, Schwartz GK, Keohan ML, et al. Progression-Free survival among patients with well-differentiated or dedifferentiated liposarcoma treated with CDK4 inhibitor Palbociclib: a phase 2 clinical trial. JAMA Oncol 2016;2:937-40.

11 Ricciotti RW, Baraff AJ, Jour G, et al. High amplification levels of MDM2 and CDK4 correlate with poor outcome in patients with dedifferentiated liposarcoma: a cytogenomic microarray analysis of 47 cases. Cancer Genet 2017;218-219:69-80.

12 Tawbi HA, Burgess M, Bolejack V, et al. Pembrolizumab in advanced soft-tissue sarcoma and bone sarcoma (SARC028): a multicentre, two-cohort, single-arm, open-label, phase 2 trial. Lancet Oncol 2017;18:1493-501.

13 Burgess MA, Bolejack V, Schuetze S, et al. Clinical activity of pembrolizumab $(\mathrm{P})$ in undifferentiated pleomorphic sarcoma (ups) and dedifferentiated/pleomorphic liposarcoma (LPS): final results of SARC028 expansion cohorts. JCO 2019;37:11015.

14 Pollack SM, He Q, Yearley $\mathrm{JH}$, et al. T-Cell infiltration and clonality correlate with programmed cell death protein 1 and programmed death-ligand 1 expression in patients with soft tissue sarcomas. Cancer 2017;123:3291-304.

15 Coindre J-M. Grading of soft tissue sarcomas: review and update. Arch Pathol Lab Med 2006;130:1448-53.

16 Robins HS, Srivastava SK, Campregher PV, et al. Overlap and effective size of the human CD8+ T cell receptor repertoire. Sci Trans/ Med 2010;2:47ra64-64.

17 Robins HS, Campregher PV, Srivastava SK, et al. Comprehensive assessment of T-cell receptor beta-chain diversity in alphabeta $\mathrm{T}$ cells. Blood 2009;114:4099-107.

18 Carlson CS, Emerson RO, Sherwood AM, et al. Using synthetic templates to design an unbiased multiplex PCR assay. Nat Commun 2013;4:2680.

19 Robins H, Desmarais C, Matthis J, et al. Ultra-Sensitive detection of rare T cell clones. J Immunol Methods 2012;375:14-19.

20 Schillebeeckx I, Armstrong JR, Forys JT, et al. Analytical performance of an immunoprofiling assay based on RNA models. $J$ Mol Diagn 2020;22:555-70.

21 Maecker HT, McCoy JP, Nussenblatt R. Standardizing immunophenotyping for the human immunology project. Nat Rev Immunol 2012;12:191-200.

22 LaFranzo NA, Flanagan KC, Quintanilha D. Predictive immune modeling of solid tumors. J Vis Exp 2020;156:e60645.

23 James NE, Miller K, LaFranzo N, et al. Immune modeling analysis reveals immunologic signatures associated with improved outcomes in high grade serous ovarian cancer. Front Oncol 2021;11:622182.

24 Harrell FE, Lee KL, Mark DB. Multivariable prognostic models: issues in developing models, evaluating assumptions and adequacy, and measuring and reducing errors. Stat Med 1996;15:361-87.

25 Moons KGM, Kengne AP, Woodward M, et al. Risk prediction models: I. Development, internal validation, and assessing the incremental value of a new (bio)marker. Heart 2012;98:683-90.

26 Tibshirani R. The LASSO method for variable selection in the COX model. Stat Med 1997;16:385-95.

27 Zou H, Hastie T. Regularization and variable selection via the elastic net. J R Stat Soc 2005;2:301-20.

28 Stromnes IM, Hulbert A, Pierce RH, et al. T-Cell localization, activation, and clonal expansion in human pancreatic ductal adenocarcinoma. Cancer Immunol Res 2017:5:978-91. 
29 Yan L, Wang Z, Cui C, et al. Comprehensive immune characterization and T-cell receptor repertoire heterogeneity of retroperitoneal liposarcoma. Cancer Sci 2019;110:3038-48.

30 Tseng WW, Malu S, Zhang M, et al. Analysis of the intratumoral adaptive immune response in well differentiated and dedifferentiated retroperitoneal liposarcoma. Sarcoma 2015;2015:1-9.

31 Petitprez F, de Reyniès A, Keung EZ, et al. B cells are associated with survival and immunotherapy response in sarcoma. Nature 2020;577:556-60.

32 Pollack SM, Redman MW, Baker KK, et al. Assessment of doxorubicin and pembrolizumab in patients with advanced
Anthracycline-Naive sarcoma: a phase $1 / 2$ nonrandomized clinical trial. JAMA Oncol 2020;6:1778-1782.

33 Allavena P, Signorelli M, Chieppa M, et al. Anti-Inflammatory properties of the novel antitumor agent Yondelis (trabectedin): inhibition of macrophage differentiation and cytokine production. Cancer Res 2005;65:2964-71.

34 Germano G, Frapolli R, Simone M, et al. Antitumor and antiinflammatory effects of trabectedin on human myxoid liposarcoma cells. Cancer Res 2010;70:2235-44.

35 Banerjee P, Zhang R, Ivan C, et al. Trabectedin reveals a strategy of immunomodulation in chronic lymphocytic leukemia. Cancer Immunol Res 2019;7:2036-51. 